\title{
THE PHYSICAL MODEL OF MAGNETIC SEPARATION IN A PLATE SEPARATOR
}

\author{
M. BROŻEK \\ University of Mining and Metallurgy, Al. Mickiewicza 30, \\ 30-059 Cracow, Poland
}

(Received 27 May 1998; Accepted 7 August 1998)

The results of magnetic separation depend on many factors, such as physical properties of particles of the separated mixture, magnetic intensity, particle sizes, separation conditions (constant or alternating field, dry or wet separation) and others. The formulae representing the dependence of separation results on the above mentioned factors are obtained from the model. The mathematical model presents only some general dependences of separations results on time or length of the separation path. A complete picture of the internal structure of the model is achieved by the analysis of process on the level of a single particle, including the particles interactions.

The paper presents a stochastic model of separation exemplified by a plate separator and its complement in the form of a physical model of separation. This model gives analytical formulae of the dependence of the specific length of the separation path and the content of the magnetic component in the magnetic product on the physical properties of particles, intensity of magnetic field and particle size. The experimental verification of the dependence of the specific length of the separation path on the particle size of mixture was given.

Keywords: Physical model; Stochastic model; Plate separator; Dry separation; Alternating field; Specific separation path

\section{INTRODUCTION}

Magnetic separation, due to the necessity of liberation of respective rock components, is carried out after relatively deep, process of crushing and milling. The separation of particles measuring a few tenths of millimeter and finer becomes very difficult since in spite of external forces acting on respective particles there are also internal forces of interactions of particles. These comprise of magnetic and surface forces. The interactions of 
particles cause the effect of flocculation. The results of magnetic separation will depend on the rate of intensity of this phenomenon.

The quantity of interactions is affected principally by the size of feed particle, physical and physicochemical properties of respective components of feed, intensity of magnetic field and separation conditions (constant or alternating field).

In order to obtain better enrichment indexes, the length of the separation path, i.e. the path of retention of the separated material in the magnetic field zone, is increased. The length of the separation path depends on the above mentioned factors. Its mathematical form can be obtained from the process model.

A purely mathematical approach to modelling does not enable a functional dependence of the length of the separation path on the values affecting significantly the process course to occur. The functional dependences are obtained from the physical model. The physical model, through the analysis of phenomena on the level of a single particle, enables the analytical dependence of the length of the separation path on the variables affecting the process, to be obtained.

So far, there have been no physical models of the magnetic separation process, taking into consideration the particles interactions. As there are many construction types of magnetic separators and the course conditions of the process reflect the specific features of each separator, the complete physical model of the process of magnetic separation should be associated with a given separator type.

This paper shows the physical model of the separation process as exemplified by a plate separator. The physical model was preceded by the description of a principle of separation in the plate separator, specification of particles interactions and the stochastic model of separation in this separator.

\section{PLATE SEPARATOR}

In a magnetic plate separator on the surface of a flat plate, the threephase current winding generates the magnetic field, variable in time and moving along the plate [1,2]. Separators with alternating magnetic field are applied to dry separation of very fine materials. This is one of the main advantages of this separator. 
Magnetic separation is accompanied by the effect of magnetic flocculation. It is especially intense in the case of separation of strongly magnetic ores, such as magnetite ore, of the granulation below $0.06 \mathrm{~mm}$. In the structure of flocs, by means of mechanical, magnetic and surface forces, the non-magnetic particles of waste rock or other minerals of low magnetic susceptibility are kept between the magnetic particles. Consequently, this leads to the deterioration of quality of the obtained magnetic concentrate.

The application of separators with alternating magnetic field causes a periodical disintegration and a change of magnetization of flocs. During this process the falling out of non-magnetic particles occurs, resulting consequently in the improvement of the concentrate quality.

The distribution of magnetic field and magnetic force, acting on the particle volume unit on the surface of the plate, is obtained by means of solving Maxwell's system of equations [3,4]:

$$
\begin{gathered}
H_{x}=\frac{j}{k} \mathrm{e}^{-k y} \cos (\omega t-\sqrt{2} k x) \\
H_{y}=\sqrt{2} \frac{j}{k} \mathrm{e}^{-k y} \sin (\omega t-\sqrt{2} k x) \\
F_{x}=\frac{2 \pi a^{3} \sqrt{2} j^{2}}{3 k} \mu_{0} \chi \mathrm{e}^{-2 k y} \sin 2(\omega t-\sqrt{2} k x) \\
F_{y}=-\frac{4 \pi a^{3} j^{2}}{3 k} \mu_{0} \chi \mathrm{e}^{-2 k y}\left[1+\sin ^{2}(\omega t-\sqrt{2} k x)\right]
\end{gathered}
$$

where $H_{x}$ and $H_{y}$ are the horizontal components of magnetic intensity, parallel and perpendicular to the plane of the separator plate, respectively; $F_{x}$ and $F_{y}$ the analogical components of magnetic force, acting on a particle of radius $a ; t$ time; $\omega$ the angular frequency; $k$ the constant, interpreted as the length of the wave vector whose value depends on the construction of the separator plate; $j$ the linear density of the plate winding current.

The component of the magnetic field vector, parallel to the plate surface and moving along the plate with a definite speed, generates the movement of magnetic flocs in the direction opposite to the field movement [5]. The falling out of non-magnetic particles takes place on 
the path of movement of flocs, due to the above mentioned periodical process of deflocculation. Since the time of deflocculation is short and the inertia of particles high, the flow of the fluidized layer of magnetite on the plate surface is observed.

The intensity of the process of the falling out of non-magnetic particles depends on many factors, such as particle size distribution of feed, frequency of the alternating field and the subsequent frequency of deflocculation as well as the speed of motion of flocs, magnetic intensity on the plate surface and, finally, magnetic susceptibility of both magnetic and non-magnetic components.

The presented physical model of the separation process in the plate separator contributes to determination of the length of the specific separation path, depending on the factors mentioned above.

\section{FORCES ACTING ON A PARTICLE AND PARTICLE INTERACTIONS}

A mineral particle in the working space of the magnetic separator is subjected to numerous forces, of different values and range of action. These forces can be divided into two groups. The first group comprises external forces under the effect of which a single mineral particle, during the absence of particle interactions, is attributed to one of two separation products, i.e. magnetic or non-magnetic. These forces comprise the magnetic force and the set of competing forces, including the gravitational, hydrodynamic and centrifugal forces.

In the conditions of magnetic separation two cases are possible which can be written as follows:

$$
\begin{aligned}
& F>\sum_{i} F_{i}^{c} \\
& F<\sum_{i} F_{i}^{c}
\end{aligned}
$$

where $\sum F_{i}^{c}$ denotes the sum of competing forces while $F$ the magnetic force. The particle gravity is a competing force in the plate separator.

In the first case, (3a), the particle will be attributed to the magnetic product. Such a particle is conventionally called a magnetic particle and 
its magnetic susceptibility is marked as $\chi_{\mathrm{m}}$. In the second case, (3b), the particle will be attributed to the non-magnetic product and is conventionally called a non-magnetic particle while its susceptibility is marked as $\chi_{\mathrm{n}}$. In case when the two forces are equal, it will be the socalled partition particle, i.e. the particle of such magnetic susceptibility that the probability of being attributed to either separation product is the same and equals 0.5 . Such a picture would exist if there were no particle interactions.

Another group of forces is composed of particles interactions, causing the phenomenon of flocculation. These interactions comprise magnetic and surface actions. Magnetic interactions consist of an interaction between two magnetic particles and between a magnetic and a nonmagnetic particle. Due to the former one, non-magnetic particles of very low susceptibility are kept within the floc volume.

Svoboda treats the magnetic interaction between two particles as a dipole-dipole interaction which, for two spheres distant by $r$ from one another, is expressed by the formula [6,7]:

$$
V_{\mathrm{m}}=\frac{1}{\mu_{0} r^{3}}\left[\vec{\mu}_{1} \cdot \vec{\mu}_{2}-\frac{3\left(\vec{\mu}_{1} \cdot \vec{r}\right)\left(\vec{\mu}_{2} \cdot \vec{r}\right)}{r^{2}}\right]
$$

where $\vec{\mu}_{1}$ and $\vec{\mu}_{2}$ are magnetic moments of particles.

For two spherical particles of radius $a$ and identical magnetic properties the expression (4) is of the form:

$$
V_{\mathrm{m}}=-\frac{32 \pi^{2} \mu_{0} \chi_{\mathrm{m}}^{2} a^{6} H^{2}}{9 r^{3}}
$$

If a non-magnetic particle (of radius $a$ ) is placed between two magnetic particles (of the same radius $a$ ), it will be kept mechanically between these particles, due to the magnetic interaction of magnetic particles. In such a situation the distance between the centres of magnetic particles will be $4 a$ and the magnetic interaction will be:

$$
V_{\mathrm{m}}=-\frac{\pi^{2} a^{3} \chi_{\mathrm{m}}^{2} \mu_{0} H^{2}}{18}
$$

The interaction between magnetic and non-magnetic particles is expressed by the formula which is similar to formula (5):

$$
V_{\mathrm{m}-\mathrm{n}}=-\frac{32 \pi^{2} \mu_{0} a^{6} \chi_{\mathrm{m}} \chi_{\mathrm{n}} H^{2}}{9 r^{3}} .
$$


At the minimum proximity of the magnetic and non-magnetic particle $r=2 a$, and expression (7) assumes the form:

$$
V_{\mathrm{m}-\mathrm{n}}=-\frac{1}{2} \pi^{2} \mu_{0} a^{3} \chi_{\mathrm{m}} \chi_{\mathrm{n}} H^{2} .
$$

Magnetic separation in the plate separator is an operation carried out in air-dry state of the enriched material. In this situation London-Van der Waals dispersive forces are particles surface interactions. According to Hamaker [8], London-Van der Waals interaction between two spherical particles of identical radii is expressed by the formula:

$$
V_{A}=-\frac{A}{6}\left[\frac{2 a^{2}}{h^{2}+4 a h}+\frac{2 a^{2}}{h^{2}+4 a h+4 a^{2}}+\ln \frac{h^{2}+4 a h}{h^{2}+4 a h+4 a^{2}}\right]
$$

where $A$ is the Hamaker's constant, $h$ the distance between two interacting surfaces.

In the case of spherical particles whose radius is much larger than the distance between their surfaces $(a \gg h)$, an approximated formula is obtained:

$$
V_{A}=-\frac{A a}{12 h}
$$

The dispersive interaction of two particles in the gaseous phase is much stronger than in liquids, due to a higher value of Hamaker's constant.

At the end of this chapter it should be stressed that the external forces separate a mixture of mineral particles into subsets which differ by their magnetic properties. On the other hand, the particle interactions hinder this process. The efficiency of the process of magnetic separation depends on the value of the latter ones.

\section{A STOCHASTIC MODEL OF THE SEPARATION PROCESS}

Figure 1 presents schematically a plate of the separator in the system of rectangular coordinates. The lined area presents a fluidized layer of magnetite and non-magnetic particles, flowing on the surface of the plate, along the $x$-axis. During the motion the non-magnetic particles fall out from this layer, forming the non-magnetic product. 


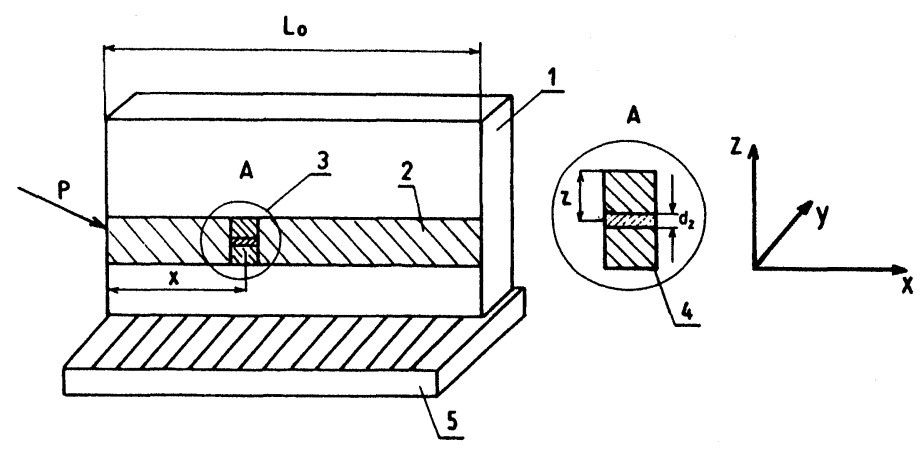

FIGURE 1 Diagram of the plate of separator: 1-plate, 2-stream of magnetic particles, 3 -fragment of the main stream, moving along the $x$-axis, 4-fragment moving along the $z$-axis, 5-containers for the non-magnetic product.

The separation of non-magnetic particles of the narrow range of physical $(\chi, \chi+\Delta \chi),(\rho, \rho+\Delta \rho)$ and geometrical $(a, a+\Delta a)$ properties will be analysed.

The process of falling out of non-magnetic particles from a floc is a random process. The number of particles which fell out from floc and were transfered into the non-magnetic product constitutes a random variable. Since the total amount of the separated non-magnetic component depends on the length of the separation path, therefore the value of the random variable will depend on the length of the separation path.

Let $x$ denote the length of the separation path while $w(x)$ be a random variable representing the number of particles of the non-magnetic component, separated up to the point $x>0$. At a variable $x$ we obtain a numerable stochastic process $\{w(x)\}$ with a continuous parameter $x$. The non-magnetic particle which fell out from the stream of material moving on the plate is a new particle. At the same time, the non-magnetic particle cannot return to the primary stream. Because of that, the process considered will be a pure birth process [10].

The conditional probability of the fact, that if $j$ particles of the nonmagnetic component were separated up to the point $x$, consequently $n$ particles of this component will be separated up to the point $x+\Delta x$ is presented by the following equation:

$$
P_{j n}(x, x+\Delta x)=P[w(x+\Delta x)=n \mid w(x)=j], \quad 0 \leq x \leq x+\Delta x .
$$


The probability function, at the assumption that the process $\{w(x)\}$ is time-homogeneous, i.e. $P_{j n}(x, x+\Delta x)=P_{j n}(\Delta x)$, fulfils the following conditions [11]:

$$
P_{j n}(\Delta x)= \begin{cases}\lambda(j) \Delta x+0(\Delta x), & \text { for } n=j+1 \\ 1-\lambda(j) \Delta x+0(\Delta x), & \text { for } n=j \\ 0(\Delta x), & \text { for } n \neq j, j+1\end{cases}
$$

Condition (12a) denotes that the probability of separating one particle at a small distance $\Delta x$ is, with the accuracy up to the infinitesimal of the higher order, proportional to the length of this section. In other words, non-magnetic particles are separated as single particles.

Condition (12b) denotes a probability that on section $\Delta x$ no nonmagnetic particle was separated. On the other hand, according to condition (12c), the probability of separating two particles on a small section $\Delta x$ is the infinitesimal of the order higher than $\Delta x$.

Function $\lambda(n)$ in formulae (12) is called the intensity function of the separation process. Assuming that $w(0)=0$, we can mark $P_{0 n}=P_{n}$. The probability $P_{n}(x)$ of the fact that $n$ particles will transfer to point $x$ to the non-magnetic product is fulfilled by the following system of equations [12]:

$$
\frac{\mathrm{d} P_{n}(x)}{\mathrm{d} x}=-\lambda(n) P_{n}(x)+\lambda(n-1) P_{n-1}(x) \quad n=1,2, \ldots, N
$$

where $N$ is the number of non-magnetic particles in the feed (in point $x=0$ ).

It was assumed for the separation intensity function $\lambda(n)$ that it fulfils the following condition:

$$
\lambda(n)=\mu(N-n)
$$

where $\mu$ is a positive constant, called the separation rate, while $(N-n)$ presents the number of non-magnetic particles, remaining in the magnetic product at point $x$ of the separation path. The value of the separation rate depends on the value of particle interactions and external forces, acting on the particle.

Condition (14) means that the intensity of the separation process of non-magnetic particles decreases with the growth of the distance from the point of material feeding. Introducing expression (14) into the system 
of equations (13) we obtain:

$$
\frac{\mathrm{d} P_{n}(x)}{\mathrm{d} x}=-\mu(N-n) P_{n}(x)+\mu[N-(n-1)] P_{n-1}(x) \quad n=1,2, \ldots, N .
$$

At the boundary condition

$$
P_{n}(0)= \begin{cases}1 & \text { for } n=0 \\ 0 & \text { for } n=1,2, \ldots, N\end{cases}
$$

the system of equations (15) has the following univocal solution [12]:

$$
P_{n}(x)=\left(\begin{array}{l}
N \\
n
\end{array}\right) \mathrm{e}^{-\mu x(N-n)}\left(1-\mathrm{e}^{-\mu x}\right)^{n} \quad \text { for } n \leq N .
$$

The expected value of the random variable $\{w(x)\}$ is:

$$
E\{w(x)\}=\sum_{n=0}^{N} n P_{n}(x) .
$$

Calculating this value by means of the generating function the following expression is obtained [12]:

$$
E\{w(x)\}=N\left(1-\mathrm{e}^{-\mu x}\right) .
$$

The following quotient:

$$
\frac{E\{w(x)\}}{N}=\varepsilon(x)=1-\exp (-\mu x)=1-\exp (-x / L)
$$

represents the recovery of the non-magnetic component in the nonmagnetic product. The value $L=1 / \mu$ presents the specific length of the separation path. This is such a length of the separation path after which the recovery of the separated non-magnetic component is equal 0.63 .

The specific length of the separation path is a function of many variables, namely, particle size $a$, density of non-magnetic particles $\rho_{\mathrm{n}}$, magnetic susceptibility of non-magnetic particles $\chi_{\mathrm{n}}$, magnetic susceptibility of magnetic particles $\chi_{\mathrm{m}}$, intensity of magnetic field $H$, frequency of 
magnetic field $\omega$, surface properties of particles $A$ and the construction parameter of the separator plate $k$. Therefore it can be written

$$
L=L\left(a, \rho_{\mathrm{n}}, \chi_{\mathrm{n}}, \chi_{\mathrm{m}}, H, \omega, A, k\right) .
$$

In order to find an apparent form of this function, it is necessary to consider the separation process in the plate separator on the level of a single particle together with taking into account the particles interactions. The next chapter will present such a physical model of separation.

\section{PHYSICAL MODEL OF THE SEPARATION PROCESS}

Figure 5 presents a rectangular fragment of the fluidized material layer, flowing on the surface of the separator plate. This fragment moves with a uniform motion towards the $x$-axis with the velocity $v_{x}$. At the same time, the infinitesimal band $\mathrm{d} z$ moves downwards with the velocity $v_{z}$. From this fragment the non-magnetic particles leave the floc, moving downwards towards $z$-axis, in the infinitesimal band $\mathrm{d} z$ under the effect of gravity.

Let the total potential of interaction of a single non-magnetic particle with the surrounding particles be equal $V_{\mathrm{c}}$. If a volume unit of the infinitesimal band $\mathrm{d} z$, after travelling the section $z$, contains $n$ non-magnetic particles, the total energy of interaction of non-magnetic particles with the surrounding is expressed as

$$
E(z)=n(z) V_{\mathrm{c}}
$$

The component $z$ of the force acting on the non-magnetic particle will be expressed by the formula

$$
F_{z}=\frac{\mathrm{d} E(z)}{\mathrm{d} z}=V_{\mathrm{c}} \frac{\mathrm{d} n(z)}{\mathrm{d} z} .
$$

In this formula the interaction potential is not subjected to differentiation because it does not depend on the variable $z$. On the other hand, however, the number of non-magnetic particles depends on the variable $z$ because, in the course of the separation process, the infinitesimal band $\mathrm{d} z$, moving along the $z$-axis, loses non-magnetic particles. 
Apart from potential forces, the non-magnetic particle, moving downwards along the $z$-axis, is affected by the force of friction with the surrounding particles. It was assumed that the force of thrust on the nonmagnetic particle is, as far as its value is concerned, equal to the force $F_{z}$, expressed by the formula (21). In this situation the force of friction will be

$$
F_{T}=\nu V_{\mathrm{c}} \frac{\mathrm{d} n(z)}{\mathrm{d} z}
$$

where $\nu$ is the friction coefficient.

The interaction expressed by the potential $V_{\mathrm{c}}$ and the friction force prevent non-magnetic particles from falling out of the floc volume. Gravity is a force causing the falling out of non-magnetic particles from the floc volume. Gravity per volume unit is:

$$
F=-m g n(z)
$$

In the conditions of equilibrium the sum of forces (21) and (22) is equal to force (23). Therefore

$$
-m g n(z)=V_{\mathrm{c}} \frac{\mathrm{d} n(z)}{\mathrm{d} z}+\nu V_{\mathrm{c}} \frac{\mathrm{d} n(z)}{\mathrm{d} z} .
$$

In the above formulae $m$ denotes the mass of a non-magnetic particle while $g$ is a acceleration of gravity. After transforming expression (24) we obtain:

$$
\frac{\mathrm{d} n(z)}{\mathrm{d} z}+\frac{m g}{V_{\mathrm{c}}(1+\nu)} n(z)=0 .
$$

Solving equation (25) we obtain an expression for a number of nonmagnetic particles which are included in a volume unit of the infinitesimal band $\mathrm{d} z$ after travelling section $z$ :

$$
n(z)=C \exp \left(-\frac{m g}{V_{\mathrm{c}}(1+\nu)} z\right)
$$

where $C$ is a positive constant determined from the condition $n(z=0)=N_{0}$, while $N_{0}$ is a number of non-magnetic particles in the feed. From the above condition $C=N_{0}$. 
The number of non-magnetic particles which left the infinitesimal band from level 0 to level $z$ is expressed as

$$
\Delta n(z)=N_{0}\left[1-\exp \left(-\frac{m g}{V_{\mathrm{c}}(1+\nu)} z\right)\right] .
$$

These particles were transfered to the non-magnetic product in section $0-x$. As it has been already mentioned before, non-magnetic particles in the floc, during the work of the separator, perform a resultant motion: towards the $z$-axis and together with the entire floc towards the $x$-axis. The floc will cover section $x$ in the time when the infinitesimal band moves by a section $z$. Therefore the following dependence is obtained:

$$
z=\frac{v_{z}}{v_{x}} x
$$

After taking into consideration dependence (28), the expression (27) assumes the form

$$
\Delta n(x)=N_{0}\left[1-\exp \left(-\frac{m g}{V_{\mathrm{c}}(1+\nu)} \frac{v_{z}}{v_{x}} x\right)\right] .
$$

Expression (29) presents the number of non-magnetic particles, separated into the non-magnetic product in section $0-x$.

If it is assumed that the velocity of motion of the floc is proportional to the velocity of the wave of the magnetic field along the plate, then $v_{x}=b \omega / k$ (where $\omega$ is the angular frequency of the magnetic field, $k$ is the length of the wave vector, depending on the separator construction, $b$ is the constant).

The recovery of the non-magnetic component into the non-magnetic product is

$$
\varepsilon_{x}=\frac{\Delta n(x)}{N_{0}}=1-\exp \left(-\frac{m g}{V_{\mathrm{c}}(1+\nu)} \frac{v_{z} k}{b \omega} x\right) .
$$

Comparing expressions (30) and (18) we obtain a formula for the specific length of the separation path

$$
L=\frac{b V_{\mathrm{c}} \omega(1+\nu)}{m g v_{\Xi} k} .
$$


It can be seen from formula (31) that the larger are the particles interactions, the more difficult it is for the non-magnetic particles to leave the floc and the larger has to be the specific length of the separation path. Similarly, with the growth of the frequency of the field, the deflocculation periods are short and the non-magnetic particles, due to their inertia, leave the floc with a delay, which must lead to the increase of the length of the separation path.

In order to make the specific length of the separation path dependent on the values mentioned in formula (19), the formulas determining the value of interactions between non-magnetic and magnetic particles should be substituted instead of $V_{\mathrm{c}}$, as well as the value of interaction of the non-magnetic particle with the magnetic field $V_{H}$, whose potential is [13]

$$
V_{H}=-\frac{2}{3} \pi \mu_{0} a^{3} \chi_{\mathrm{n}} H^{2} .
$$

After substituting all the above interactions into formula (31) we obtain the required dependence of the specific separation path on all variables, mentioned in formula (19):

$L=\frac{\left(A / 12 a^{2} r+\frac{1}{18} \pi^{2} \mu_{0} \chi_{\mathrm{m}}^{2} H^{2}+\frac{1}{2} \pi^{2} \mu_{0} \chi_{\mathrm{m}} \chi_{\mathrm{n}} H^{2}+\frac{2}{3} \pi \mu_{0} \chi_{\mathrm{n}} H^{2}\right) \omega b(1+\nu)}{\frac{4}{3} \pi \rho_{\mathrm{n}} g v_{z} k}$.

It results from formula (33) that with the increase of magnetic intensity and magnetic susceptibility of both non-magnetic and magnetic particles the specific length of the separation path increases. The length of the separation path depends strongly on particle size. The length of the separation path increases with the decrease of sizes of separated particles. It results from the fact that in the case of extremely fine particles the fraction of surface interactions grows in the general balance of particles interactions.

\section{EXPERIMENTAL}

The dependence of the specific separation path on the size of separated particles and magnetic susceptibility of non-magnetic particles was verified. 
To verify the dependence of the specific separation path on particle sizes mixtures of magnetite and sand in the following size fractions: $0.2-$ $0.16 \mathrm{~mm} ; 0.16-0.125 \mathrm{~mm} ; 0.125-0.10 \mathrm{~mm} ; 0.10-0.071 \mathrm{~mm} ; 0.056-$ $0.04 \mathrm{~mm}$; and $0.04-0.02 \mathrm{~mm}$ were prepared. The volume contents of magnetite and sand were equal to $50 \%$ of magnetite and $50 \%$ of sand.

To verify the effect of magnetic susceptibility of non-magnetic particles on the specific separation path mixtures of magnetite with the prior separated two ilmenite fractions of different magnetic susceptibility were prepared. The volume content of magnetite in mixtures was $50 \%$, while the granulation of samples was $0.2-0.16 \mathrm{~mm}$.

The samples prepared in this way were separated in the plate separator. The value of magnetic intensity on the surface of the separator plate was $58 \mathrm{kA} / \mathrm{m}$, the length of the separator plate $L_{0}=1.0 \mathrm{~m}$, frequency of the magnetic field was $50 \mathrm{~Hz}$. The non-magnetic product was collected in special containers of certain lengths, placed just under the separator plate. The masses of the non-magnetic component in respective containers were used to calculate the recovery of the non-magnetic component in the non-magnetic product and to determine the dependence of this recovery on the distance $x$ from the feeding point of material.

\section{ANALYSIS OF RESULTS}

Figure 2 presents the dependence of the recovery of the non-magnetic component (sand) in the non-magnetic product on the length of the separation zone for the tested size fractions. The experimental values were marked with points, while continuous curves represent the dependences determined by means of fitting the empirical data to the model equation (18). The specific length of the separation path $L$ is the model parameter.

Mixtures of quartz sand and magnetite were used to investigate the dependence between the specific length of the separation path and particle sizes. Pure quartz sand has diamagnetic properties. Its magnetic susceptibility is very low and reaches $-1.2 \cdot 10^{-6} \mathrm{cgs}$ [13]. Therefore, in formula (33) for the length of the specific separation path, it is possible to neglect magnetic interactions of non-magnetic particles with magnetic particles and the magnetic field. In this case the dependence of the specific separation path on particle sizes will be expressed by the following 


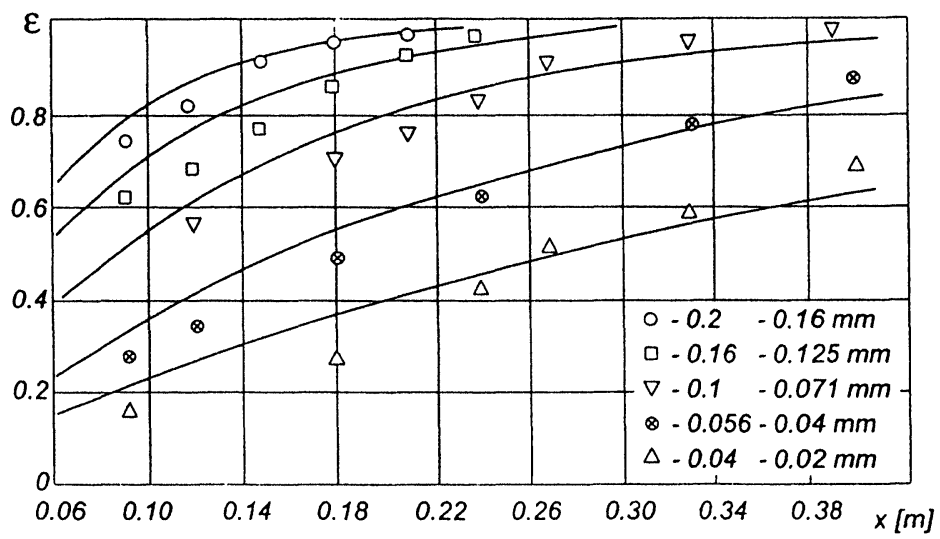

FIGURE 2 The dependence of the recovery of sand in the non-magnetic product on the length of the separation zone for different particle size.

formula:

$$
L(a)=c_{1}+\frac{c_{2}}{a^{2}}
$$

where $c_{1}$ and $c_{2}$ are constants, expressed as

$$
c_{1}=\frac{\pi \mu_{0} \chi_{\mathrm{m}}^{2} H^{2} \omega b(1+\nu)}{24 \rho_{\mathrm{n}} g v_{z} k}
$$

and

$$
c_{2}=\frac{A \omega b(1+\nu)}{16 \pi r \rho_{\mathrm{n}} g v_{z} k} .
$$

Figure 3 presents the dependence of the specific separation path on the feed particle size. The continuous line represents a curve of the following equation:

$$
L=0.05+\frac{0.00032}{a^{2}}
$$

where $a$ is expressed in millimeters while $L$ in metres. The values of the specific separation path, calculated according to Fig. 2, are marked with circles in Fig. 3. As it can be seen from Fig. 3, the Eq. (35) approximates 


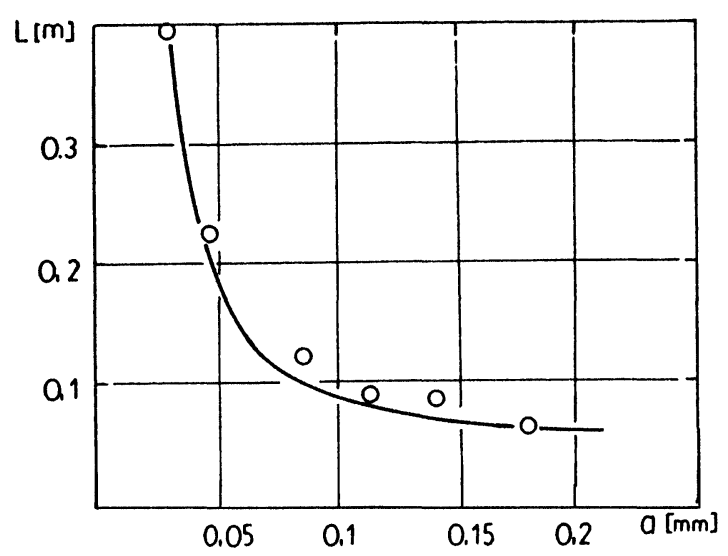

FIGURE 3 The dependence of the specific length of the separation path on the particle size.

well the dependence of the specific separation path on the particle size, in dry separation. Rapid growth of the length of the separation path occurs for the particles below $75 \mu \mathrm{m}$.

Due to a significant growth of the specific separation path together with the decrease of particle size, dry separation is performed for loose materials, deprived of the finest particles.

Figure 4 presents the dependences, experimental and model ones, of the recovery of the non-magnetic component in the non-magnetic product on the length of the separation zone for two values of magnetic susceptibility of non-magnetic particles.

As it can be seen from the above figure, the specific length of the separation path grows with the increase of magnetic susceptibility of non-magnetic particles. This experimental result is compatible with formula (33) from which it results that the value of interactions of nonmagnetic particles with magnetic ones and the magnetic field grows with the increase of magnetic susceptibility of non-magnetic particles, which hinders the falling out of non-magnetic particles from the stream of magnetic product.

The difference between magnetic susceptibility of magnetic and nonmagnetic particles decreases with the increase of magnetic susceptibility of non-magnetic particles and their separation becomes more difficult. 


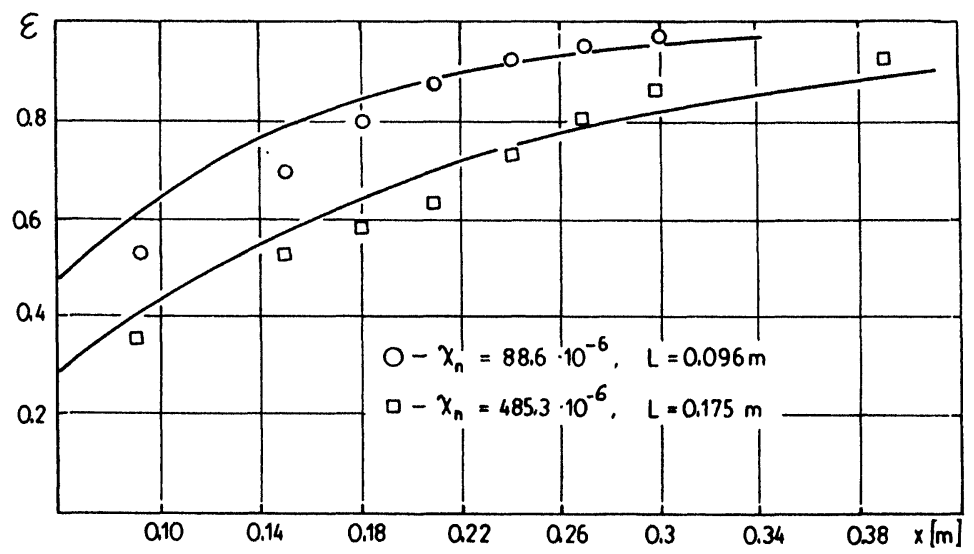

FIGURE 4 The dependence of the recovery of the non-magnetic component on the length of the separation zone for different values of magnetic susceptibility $\chi_{n}$.

This is revealed by the increase of the specific length of the separation path or multiplicity of separation in the case of multi-stage enrichment.

Specific length of the separation path can be analytically connected with the content of the magnetic component in the magnetic concentrate. The remains of the non-magnetic component in the magnetic concentrate can be expressed by formula [14]:

$$
\sigma=\frac{\gamma_{\mathrm{m}} \lambda_{\mathrm{n}}}{\alpha_{\mathrm{n}}}=\exp (-x / L)
$$

where $\gamma_{\mathrm{m}}$ is the yield of the concentrate, $\lambda_{\mathrm{n}}$ the content of the nonmagnetic component in the concentrate, $\alpha_{\mathrm{n}}$ the content of the nonmagnetic component in the feed.

When the material is separated into two products, the equation of mass balance is as follows:

$$
\alpha_{\mathrm{n}}=\gamma_{\mathrm{m}} \lambda_{\mathrm{n}}+\left(1-\gamma_{\mathrm{m}}\right) \vartheta_{\mathrm{n}}
$$

where $\vartheta_{\mathrm{n}}$ represents the content of non-magnetic component in the nonmagnetic product. With the two-component feed, the concentrate can contain the magnetic component $\left(\beta_{\mathrm{m}}\right)$ and the non-magnetic one $\left(\lambda_{\mathrm{n}}\right)$, whereas

$$
\lambda_{\mathrm{n}}+\beta_{\mathrm{m}}=1 \text {. }
$$


It is possible to calculate the content of the magnetic component in the concentrate from Eqs. (36)-(38).

$$
\beta_{\mathrm{m}}=\frac{\vartheta_{\mathrm{n}}-\alpha_{\mathrm{n}}+\alpha_{\mathrm{n}}\left(1-\vartheta_{\mathrm{n}}\right) \exp (-x / L)}{\vartheta_{\mathrm{n}}-\alpha_{\mathrm{n}}+\alpha_{\mathrm{n}} \exp (-x / L)} .
$$

When it is assumed that exclusively the non-magnetic component is transfered to the non-magnetic product $\left(\vartheta_{n}=1\right)$, then it is obtained from expression (39)

$$
\beta_{\mathrm{n}}=\left[1+\frac{\alpha_{\mathrm{n}}}{1-\alpha_{\mathrm{n}}} \exp (-x / L)\right]^{-1} .
$$

Therefore the quality of the concentrate is determined by the feed composition $\left(\alpha_{\mathrm{n}}\right)$, the length of the separation zone $x$ and the specific length of the separation path $(L)$, as well as, indirectly, by all the variables mentioned in formula (19). An apparent form of this dependence is specified by expression (40) and (33).

For the dependence $L(a)$, represented by formula (35), the dependence of the content of the magnetic component in the concentrate on the particle size, at the fixed length of the separation zone, $x=0.1 \mathrm{~m}$ and $\alpha_{\mathrm{n}}=0.5$, is as follows:

$$
\beta_{\mathrm{m}}(a)=\left[1+\exp \left(-\frac{a^{2}}{0.5 a^{2}+0.0032}\right)\right]^{-1} .
$$

The above dependence is shown in Fig. 5. The decrease of the content of the magnetic component in the concentrate at the decreasing size of separated particles results from the growth of the effect of London-Van der Waals surface interactions. For particles greater than $0.2 \mathrm{~mm}$ the particle size has no effect on the separation results.

The above model did not consider formally the electrostatic interaction, resulting from tribo-electrization of particles against the plate surface. This interaction will intensify the process of flocculation and adhesion of particles to the plate surface which, consequently, leads to increase of the length of the separation path and decrease of the content of the magnetic component in the magnetic product. The formula for the interaction force of two particles, considering the electrostatic 


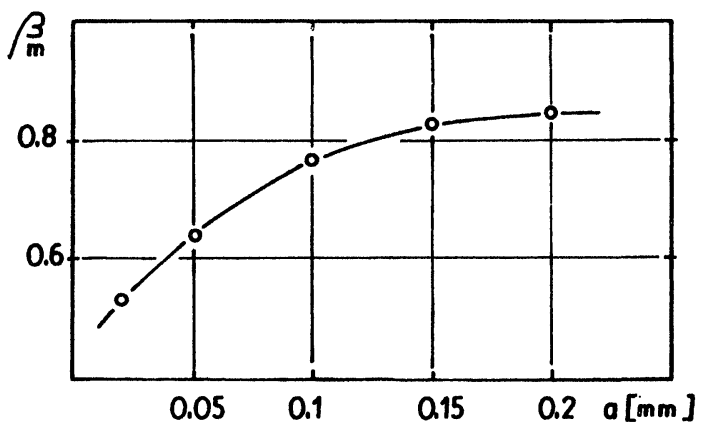

FIGURE 5 The dependence of the content of the magnetic component in the concentrate on the particle size.

interaction (as given by Derkach [14]), is analogical to formula (10) as far as the dependence on the particle size is concerned. Therefore it can be stated that dependence (35), determined experimentally, results from the resultant of London-Van der Waals and electrostatic interactions.

\section{References}

[1] W. Pilch and B. Topolnicka. Zeszyty Naukowe AGH, no. 381, 159 (1972) (in Polish).

[2] W. Pilch et al. Proc. XI IMPC, Cagliary 1975, p. 361.

[3] M. Brożek et al. Archives of Mining Sciences, 28, 509 (1983).

[4] M. Brożek et al. Proc. XVIMPC, Cannes 1985, 1, p. 435.

[5] A. Siwiec and Tran Quang Bang. Archives of Mining Sciences, 34, 345 (1989).

[6] J. Svoboda. Int. J. Min. Proces., 8, 377 (1981).

[7] J. Svoboda. IEEE Trans. Magn., 18, 796 (1982).

[8] H.C. Hamaker. Physica, 4, 1058 (1937).

[9] J.Th.G. Overbeek. Powder Technology, 37, 195 (1984).

[10] N.T.J. Bailey. The Elements of Stochastic Processes with Application to the Natural Sciences. John Wiley \& Sons, New York, London, Sydney, 1964.

[11] W. Kraj. Bulletin of Polish Academy of Science, 15, 419 (1967).

[12] J. Litwiniszyn. Bulletin of Polish Academy of Science, 11, 81 (1963).

[13] J. Svoboda. Magnetic Methods for the Treatment of Minerals. Elsevier Sci. Publ., Amsterdam, Oxford, New York, Tokyo, 1987.

[14] V.G. Derkach. Special Methods of the Beneficiation of Mineral (in Russian). Nedra, Moscow, 1966. 